\title{
Distant survival for patients undergoing surgery using volatile versus IV anesthesia for hepatocellular carcinoma with portal vein tumor thrombus: a retrospective study
}

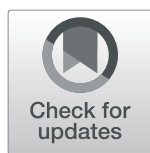

Xiao-Yan Meng ${ }^{1,2+}$, Xiu-Ping Zhang ${ }^{3+}$, Zhe Sun ${ }^{3+}$, Hong-Qian Wang ${ }^{1}$ and Wei-Feng Yu ${ }^{1,2^{*}}$ (D)

\begin{abstract}
Background: Whether anesthesia type is associated with the surgical outcome of Hepatocellular carcinoma (HCC) patients with portal vein tumor thrombus (PVTT) remains to be determined. This study aims to investigate the impact of volatile inhalational anesthesia (INHA) versus total IV anesthesia (TIVA) on the survival outcomes in HCC patients with PVTT.

Methods: A cohort of in-patients whom were diagnosed of HCC with PVTT in Eastern Hepatobiliary Surgery Hospital, Shanghai, China, from January 1, 2008 to December 24, 2012 were identified. Surgical patients receiving the INHA and TIVA were screened out. The overall survival (OS), recurrence-free survival (RFS) and several postoperative adverse events were compared according to anesthesia types.

Results: A total of 1513 patients were included in this study. After exclusions are applied, 263 patients remain in the INHA group and 208 in the TIVA group. Patients receiving INHA have a lower 5 -year overall survival rate than that of patients receiving TIVA [12.6\% (95\% Cl, 9.0 to 17.3$)$ vs. $17.7 \%(95 \% \mathrm{Cl}, 11.3$ to 20.8$), P=0.024]$. Results of multivariable Cox-regression analysis also identify that INHA anesthesia is significantly associated with mortality and cancer recurrence after surgery compare to TIVA, with HR $(95 \% \mathrm{Cl})$ of $1.303(1.065,1.595)$ and $1.265(1.040,1.539)$, respectively. Subgroup analysis suggested that in more severe cancer patients, the worse outcome related to INHA might be more significant.
\end{abstract}

Conclusion: This retrospective analysis identifies that TIVA is associated with better outcomes compared with INHA. Future prospective studies clinical and translational studies are required to verify this difference and investigate underlying pathophysiology.

Keywords: Hepatocellular carcinoma, Portal vein tumor Thrombus, Volatile inhalational anesthesia, Total IV anesthesia

\footnotetext{
* Correspondence: ywf808@sohu.com

${ }^{+}$Xiao-Yan Meng, Xiu-Ping Zhang and Zhe Sun contributed equally to this work.

${ }^{1}$ Department of Anesthesiology, Eastern Hepatobiliary Surgery Hospital, the Second Military Medical University, 225 Changhai Road, Shanghai, China ${ }^{2}$ Department of Anesthesiology, Ren Ji Hospital, School of Medicine, Shanghai Jiao Tong University, 160 Pudian Road, Shanghai, China Full list of author information is available at the end of the article
}

(c) The Author(s). 2020 Open Access This article is licensed under a Creative Commons Attribution 4.0 International License, which permits use, sharing, adaptation, distribution and reproduction in any medium or format, as long as you give appropriate credit to the original author(s) and the source, provide a link to the Creative Commons licence, and indicate if changes were made. The images or other third party material in this article are included in the article's Creative Commons licence, unless indicated otherwise in a credit line to the material. If material is not included in the article's Creative Commons licence and your intended use is not permitted by statutory regulation or exceeds the permitted use, you will need to obtain permission directly from the copyright holder. To view a copy of this licence, visit http://creativecommons.org/licenses/by/4.0/. The Creative Commons Public Domain Dedication waiver (http://creativecommons.org/publicdomain/zero/1.0/) applies to the data made available in this article, unless otherwise stated in a credit line to the data. 


\section{Background}

Volatile inhalational (INHA) and IV anesthesia (TIVA) are two methods commonly used in general anesthesia maintenance. Currently, several researches reported that INHA was associated with worse postoperative outcomes compare to INHA in certain types of cancers. Dr. Wigmore et al. [1] did a retrospective analysis which firstly compared long-term survival in more than 7000 patients undergoing elective cancer surgeries, and reported that mortality of patients accepted INHA is approximately $50 \%$ greater than those accepted TIVA. Since then, more studies reported similar results in different cancers [2]. Besides from these clinical evidences, animal researches also reported that administration of volatile inhalational agents was associated with upregulation of tumorigenic growth factors including hypoxia-inducible factors (HIFs) and insulin-like growth factor (IGF) [3, 4], which are highly associated with progression angiogenesis and cell proliferation in tumor.

Although the underlying mechanism remains unclear, these results have drawn due attention that anesthesia technique might be an independent risk factor for postoperative outcomes of most cancers, including liver cancer. Of note, previous studies also reported that the MAC of sevoflurane is lower in patients with end-stage liver cancer [5]. Thus, we hypothesize that INHA might be associated with lower 5-year overall survival (OS) compared with TIVA in hepatocellular carcinoma (HCC) patients with portal vein tumor thrombus (PVTT), an end-stage liver cancer with a high recurrence rate and reduced median survival time (MST) [6-9], in considering that in these end stage cancer patients, even subtle differences in medication might lead to significant effects on long-term outcome.

\section{Methods}

\section{Study design}

We retrospectively identified all patients who underwent aggressive surgical liver resection for selected HCC patients with PVTT at Eastern Hepatobiliary Surgery Hospital from January 1, 2008 to December 24, 2012. Exclusion criteria including: (1) no surgical treatment performed; (2) received mixed inhalational and intravenous anesthesia; (3) received additional procedures with different anesthesia or for other diseases afterwards; (4) received extra sedation in ICU or in general ward after surgery; (5) less than 18 years old; (6) had an urgent or emergence surgery and (7) incomplete follow-up data. The research was approved by the Ethics Committee of the Eastern Hepatobiliary Surgical Hospital of China. Written informed consents to record clinical follow up data were obtained from participants or their surrogates during hospitalization.
Baseline data retrospectively extracted including anesthetic technique, year of surgery, age at the time of surgery, sex, American Society of anesthesiologists' (ASA) physical status classification, pre-existing diagnosis of diabetes or hypertension, HBV surface antigen (HBsAg) and HCV anti-body (HCV-ab). Data related to patients' preoperative liver function, cytonecrosis and cancer statue were also documented, including Child-Pugh score, alpha-fetoprotein (AFP), type of PVTT, tumor diameter as well as alanine aminotransferase (ALT) and aspartate aminotransferase (AST) levels.

\section{Outcomes}

The primary outcome was 5-year OS. Secondary outcomes were (1) recurrence-free survival (RFS); (2) 30day mortality; (3) a set of major adverse cardiac events (MACE) that included myocardial infarction (MI), cardiac arrest, or newly diagnosed malignant arrhythmia; (4) multiple organ dysfunction (MOD) primarily induced of acute hepatic failure postoperatively; (5) blood loss and blood transfusion; (6) hospital length of stay (7) postoperative ALT and AST were also recorded.

\section{Anesthesia techniques}

Patients were divided based on INHA or TIVA they received for maintenance of anesthesia. Patients in the TIVA group received continuous infusions of propofol, and those in the INHA group received sevoflurane. Supplementary opioid for maintaining were used at the discretion of the anesthetist in all patients, including sufentanil and/or remifentanil, with the highest dose no more than $50 \mathrm{mg}$ and $2 \mathrm{mg}$, respectively. No other sedative-hypnotic drugs were used during maintenance.

Type of anesthesia was according to the anesthetist's decision, mainly depending on their preference and proficiency of the anesthesia technique. Details of the surgical process as previously described [10].

\section{Statistical analyses}

The Kaplan-Meier method was used to calculate the overall survival and recurrence-free survival of patients from the date of surgery to the date of events. A univariable Cox regression analysis was applied, and for variables with P less than 0.1 were then included into the multivariable model to identify risk factors.

Secondary outcomes were compared using chisquare or Mann-Whitney-Wilcoxon tests as appropriate. Missing values (all less than 5\%) were filled by the average value of the variable. Significant difference defined as $P<0.05$ in all analysis (SPSS version 22.0; IBM Inc., USA). 


\section{Results}

\section{Baseline characters and survival for all patients}

A total of 1523 patients whom are diagnosed of HCC with PVTT are delivered in the study period. After exclusions applied, 471 patients are included in the analysis, with 263 patients in the INHA group and 208 in the TIVA (Fig. 1). The mean age is 48.6 years old; The majority of patients are male (90.6\%), had a grade of ASA II (88.3\%) and Child-Pugh A (88.4\%); $410(87.0 \%)$ of patients have large hepatocellular carcinoma (> $10 \mathrm{~cm})$; 408 (86.6\%) are identified with $\mathrm{HBsAg}-+$, including 4 with both $\mathrm{HBsAg}-+$ and $\mathrm{HCV}-$ $\mathrm{ab}+$. Only 4 patients are identified with $\mathrm{HCV}-\mathrm{ab}-+$ alone, which is not enough for effective analysis. Fiveyear survival rate for all patients is $14.8 \%$ (95\% CI, 11.3 to 17.6 ), with median survival time of 9.0 month (95\% CI, 7.9 to 10.0). The patient characteristics in two groups are described in Table 1.

\section{Five-year OS and RFS}

Results of Kaplan-Meier survival analysis show that, compare with TIVA, INHA is associated with a worse 5year OS rate $[17.7 \%$ (95\% CI, 11.3 to 20.8 VS. $12.6 \%$ (95\% CI, 9.0 to 17.3$)$ ); $P=0.024$, Fig. $2 \mathrm{a}]$, as well as a worse 5-year RFS rate[15.4\% (95\% CI, 12.6 to 18.1 ) VS. $11.7 \%$ (95\% CI, 9.7 to 13.8 ); $P=0.032$, Fig. 2b]. On univariable analysis, 6 potential risk factors have $P<0.1$ are included in multivariable model (Supplementary table 1). Results of multivariable analysis also suggest that INHA is an independent risk factor for mortality [HR (95\%CI), $1.303(1.065,1.595)]$ and cancer recurrence [HR (95\% CI), 1.265 (1.040, 1.539); Table 2] in 5 years after surgery.

\section{Other secondary outcomes}

Other outcomes including 30-day mortality rate, postoperative MACE and MOF rate, as well as blood loss, blood transfusion and length of stay in hospital are similar in both groups (Table 3). Postoperative serum biomarker of ALT and AST are compared (with incomplete data), the results suggest a minor liver cytonecrosis of TIVA after surgery (Supplementary Figure 1).

\section{Subgroup analysis}

In multivariable model, four more variables are screened out as independent risk factors for 5-year OS and RFS: Child-Pugh, AFP level, diameter of hepatocellular carcinoma and PVTT type. We then did a subgroup

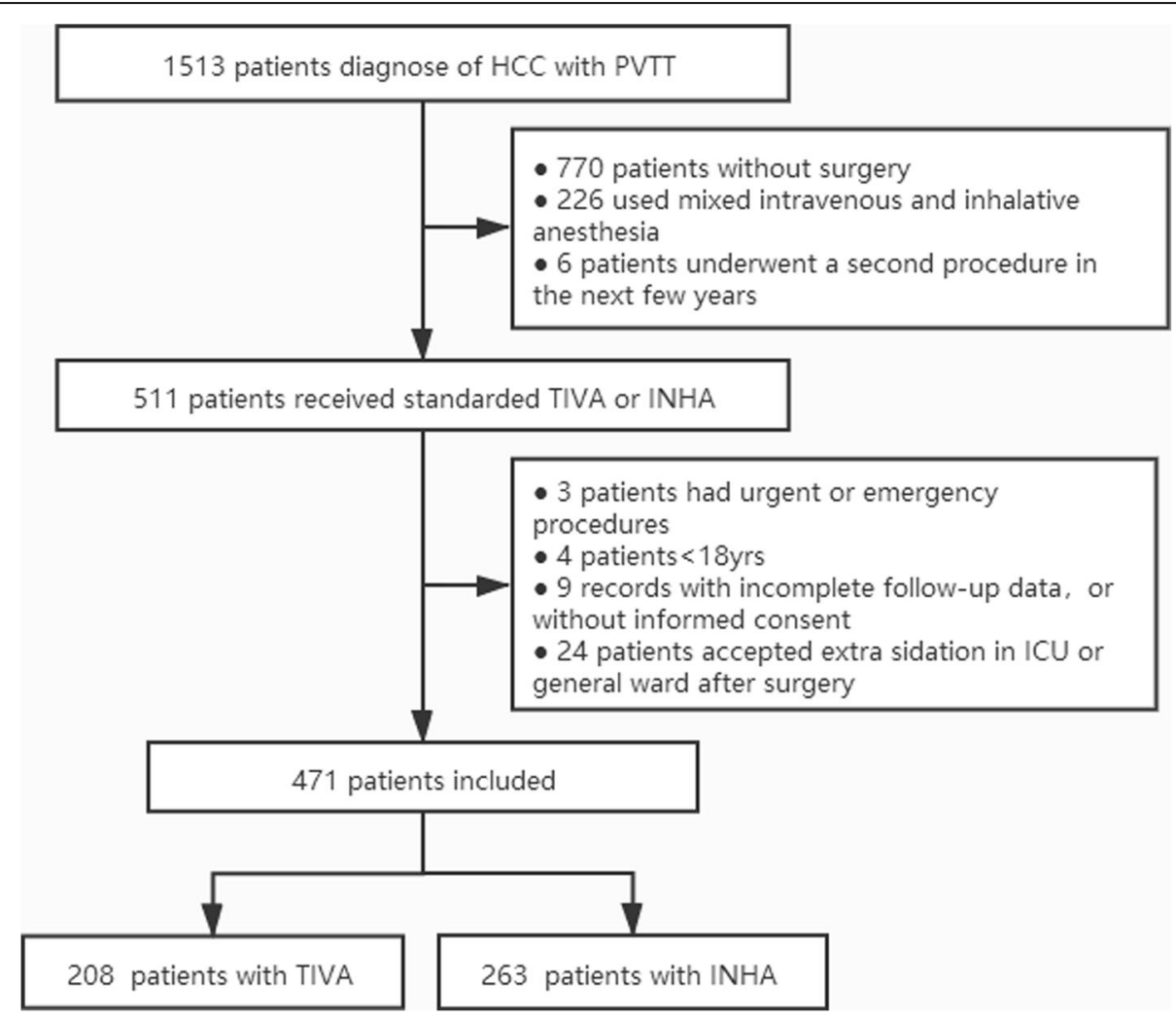

Fig. 1 Flow diagram detailing the selection of patients included in the retrospective analysis. INHA = volatile inhalational; TIVA = total IV anesthesia 
Table 1 Patient baseline characters

\begin{tabular}{|c|c|c|c|}
\hline Variables & TIVA $(N=208)$ & INHA $(N=263)$ & $P$ value \\
\hline & $\mathrm{N}(\%)$ & N (\%) & \\
\hline Sex (male) & $188(90.4)$ & $239(90.9)$ & 0.856 \\
\hline $\mathrm{HBsAg}-+^{\mathrm{a}}$ & $182(87.5)$ & $226(85.9)$ & 0.619 \\
\hline \multicolumn{4}{|l|}{ ASA } \\
\hline$\|$ & $186(89.4)$ & $229(87.1)$ & \multirow[t]{3}{*}{0.191} \\
\hline III & $22(10.6)$ & $30(11.4)$ & \\
\hline IV & $0(0)$ & $4(1.5)$ & \\
\hline \multicolumn{4}{|l|}{ Child-Pugh } \\
\hline A & $184(88.5)$ & 233 (88.6) & \multirow[t]{3}{*}{0.662} \\
\hline B & $22(10.6)$ & $25(9.5)$ & \\
\hline C & $2(0.4)$ & $5(1.1)$ & \\
\hline \multicolumn{4}{|l|}{ AFP (ug/L) } \\
\hline$<25$ & $30(14.4)$ & $54(20.5)$ & \multirow[t]{4}{*}{0.109} \\
\hline $25-399$ & 39 (18.8) & $45(17.1)$ & \\
\hline 400-999 & $12(5.8)$ & $25(9.5)$ & \\
\hline$\geq 1000$ & $127(61.1)$ & $139(52.9)$ & \\
\hline \multicolumn{4}{|c|}{ Tumor Diameter (cm) } \\
\hline$<5$ & $1(0.4)$ & $2(0.8)$ & \multirow[t]{3}{*}{0.546} \\
\hline $5-9.9$ & $22(10.6)$ & 36 (13.7) & \\
\hline$\geq 10$ & $185(88.9)$ & $225(85.6)$ & \\
\hline \multicolumn{4}{|l|}{ PVTT } \\
\hline 1 & $30(14.4)$ & $40(15.3)$ & \multirow[t]{3}{*}{0.716} \\
\hline 2 & $131(63.0)$ & $163(62.0)$ & \\
\hline 3 & $47(22.1)$ & $60(22.8)$ & \\
\hline \multicolumn{4}{|c|}{ Year of surgery } \\
\hline 2008 & $70(33.7)$ & $79(30.0)$ & \multirow[t]{6}{*}{0.882} \\
\hline 2009 & $33(15.9)$ & $42(16.0)$ & \\
\hline 2010 & $31(14.9)$ & $46(17.5)$ & \\
\hline 2011 & $31(14.9)$ & $37(14.1)$ & \\
\hline \multirow[t]{2}{*}{2012} & $43(20.7)$ & $59(22.4)$ & \\
\hline & Mean (SD) & Mean (SD) & \\
\hline \multirow[t]{2}{*}{ Age (yr) } & $48.0(10.94)$ & $49.0(9.73)$ & \multirow[t]{2}{*}{0.078} \\
\hline & Median (IQR) & Median (IQR) & \\
\hline WBC $\left(10^{9} / L\right)$ & $5.5(4.2,7.3)$ & $5.4(4.3,7.1)$ & 0.06 \\
\hline ALT (U/L) & $47.8(31.0,66.0)$ & $45.0(29.1,67.3)$ & 0.903 \\
\hline AST (U/L) & $49.0(37.0,69.0)$ & $52.0(35.0,71.0)$ & 0.820 \\
\hline
\end{tabular}

ASA American Society of Anesthesiologists, PVTT Portal vein tumor thrombus, INHA Volatile inhalational anesthesia, TIVA Total IV anesthesia, WBC White blood cells, NLR Neutrophil-lymphocytes ratio

${ }^{\mathrm{a}}$ Including 4 patients with $\mathrm{HBsAg}-+$ and anti-HCV - +

Kaplan-Meier survival analysis to estimate the association of anesthesia type on postoperative OS and RFS in different sub-variable groups, there were Child-Pugh A; Child-Pugh B\&C; tumor diameter $<10 \mathrm{~cm}$; tumor diameter $\geq 10 \mathrm{~cm} ;$ AFP $<400 \mu \mathrm{g} / \mathrm{L} ;$ AFP $\geq 400 \mu \mathrm{g} / \mathrm{L} ;$ PVTT typeI; PVTT typeII; PVTT typeIII (Figs. 3, 4, 5, and 6). The results suggest that INHA was associated with significant lower OS and RFS rate compare with TIVA in several sub-variable groups indicating more severe liver cancer status, including tumor diameter $\geq 10 \mathrm{~cm}$ (Fig. 4b, d); AFP $\geq 400 \mu \mathrm{g} / \mathrm{L}$ (Fig. 5b, d); PVTT typeIIand PVTT typeIII (Fig. 6c, e, f).

\section{Discussion}

This retrospective analysis evaluates long-term OS, RFS and several short-term postoperative adverse events in 1513 HCC patients with PVTT receiving INHA and TIVA. We identify that patients receiving INHA using sevoflurane had a lower 5-year OS and RFS rate than that of patients receiving TIVA using propofol. On multivariable Cox regression analysis, we identify that INHA is an independent risk factor for mortality and cancer recurrence in 5-year after surgery. In subgroup analysis, our results suggest that patients accepted INHA, compare with those who accepted TIVA, have worse survival rates when there are in severe liver cancer status. No significant differences in postoperative adverse events and 30-day mortality are found between the two groups in this study.

Clinical evidence of anesthesia type on surgical outcomes Recently, several clinical studies have been investigated for anesthesia type on postoperative outcomes in elective cancer patients. Enlund et al. [6] did a retrospective analysis based on 2838 patients with breast, rectal, and colon cancer, they reported that the overall survival for patients receiving propofol anesthesia is $4.7 \%$ higher at $1-y r$ and $5.6 \%$ higher at 5 -yr than those receiving sevoflurane. But after balance for confounders, this differences are not significant. In another study, Wigmore et al. [1] analyzed 7030 patients who underwent elective cancer surgery over a 3-yr period, They reported a worse outcome in patients receiving volatile anesthesia, with a $\mathrm{HR}$ of 1.46 (95\% CI, 1.29 to 1.66) for death, compare with TIVA. In addition, Yan et al. [7]. designed a randomized controlled trail in 80 breast cancer patients, they reported that the total intravenous anesthesia can inhibit the release of vascular endothelial growth factor $\mathrm{C}$ (VEGF-C) in breast surgery, yet with no significant benefice in the short-term recurrence rate of breast cancer. Importantly, a recent meta-analysis with 9 retrospective studies and 1 RCT concluded that, the use of TIVA was associated with improved RFS in all cancer types and improved OS in several certain types of cancers [2]. These studies have achieved consistent results that TIVA anesthesia has a better long-term 


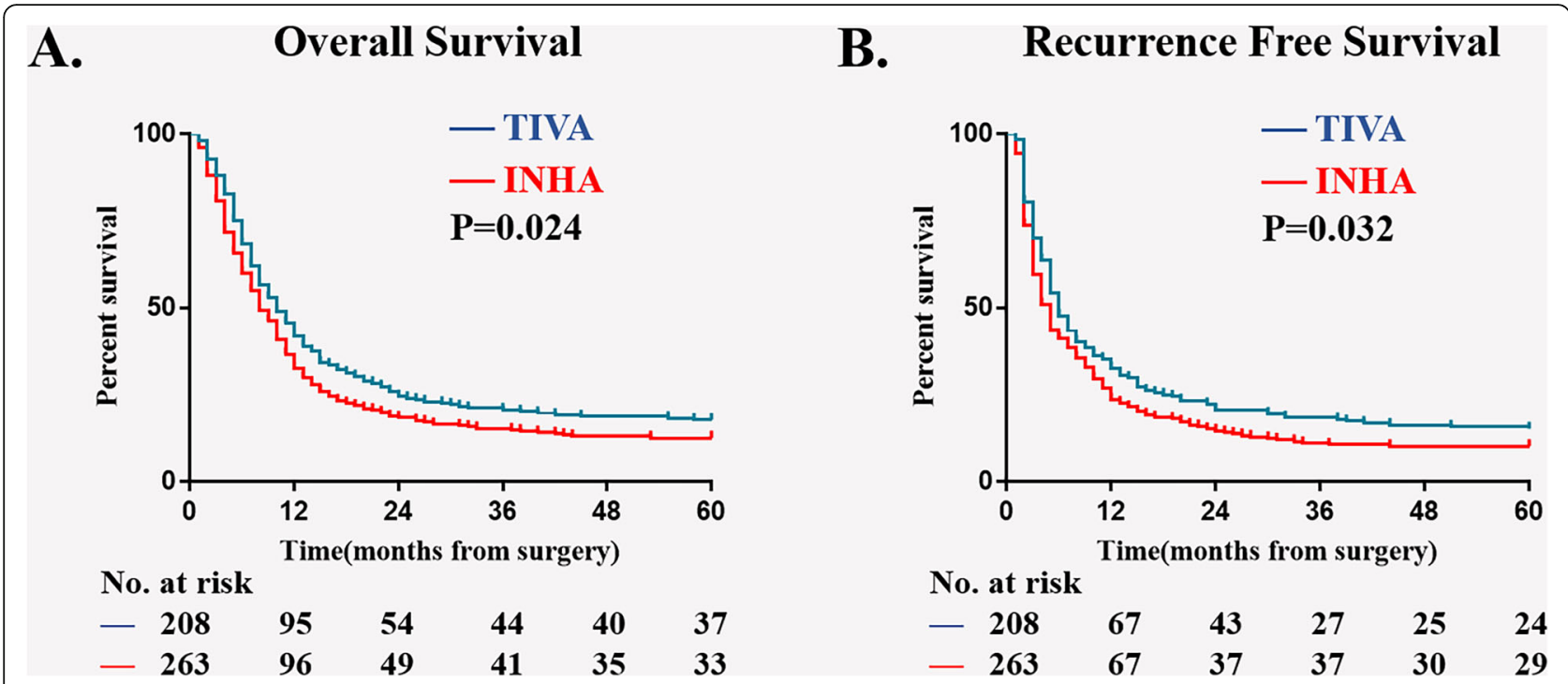

Fig. $\mathbf{2}$ Kaplan-Meier survival curves from the date of surgery by anesthesia type for (a) overall survival in patients before matching $(P=0.007)$, $(\mathbf{b})$ overall survival in patients after matching $(P=0.044)$, (c) recurrence-free survival in patients before matching $(P=0.020)$, (d) recurrence-free survival in patients after matching $(P=0.081)$. INHA = volatile inhalational; TIVA = total IV anesthesia

prognosis for patients undergoing tumor resection compared with INHA. However, the population these studies enrolled varies a lot. Yet meaningful conclusions on whether TIVA is superior to INHA for all cancer patients or just for certain types of cancer are difficult to define. To this end, the current study sort to compare the long-term survival rate in TIVA and INHA in HCC patients with PVTT.

\section{Laboratory evidence of anesthetics on tumors metastasis and recurrence}

Over the years, numerous animal and laboratory studies have been investigated for the mechanism of anesthetic agents on primary tumors metastasis and recurrence. Cellular immune system and tumor proliferation-associated factors are considered to play a key role in it. For instance, propofol has been demonstrated to have a preservation effective on $\mathrm{T}$ lymphocyte activity and Th1 cytokine secretion, or even inhibits tumor growth in animal model $[8,9$, 11]. Of note, $\mathrm{T}$ lymphocytes and NK cells are two major cytotoxic effector cells that participate in cellmediated immune responses. Meanwhile, researches also proved that sevoflurane could inhibit primary leukocyte integrin lymphocyte function and could induced lymphocyte apoptosis through downregulation of Lymphocyte Function-associated Antigen 1 (LFA1 ), thus promoting tumor recurrence and metastasis [12]. Moreover, studies both in vivo [13] and in patients undergoing breast cancer surgery [14] have reported an inhibitory effect of anesthetic agents on natural killer cell function, and further promotes tumor recurrence. This inhibitory effect is probably related to the dysfunction in CD16 cell and CD107 $\alpha$ NK receptor after exposure to sevoflurane [15]. More recently, Bellanti et al. [16] demonstrated that

Table 2 Cox proportional hazard regression analyses: multivariable model for overall survival and recurrence-free survival

\begin{tabular}{|c|c|c|c|c|}
\hline \multirow[t]{2}{*}{ Variables } & \multicolumn{2}{|l|}{ Overall Survival } & \multicolumn{2}{|c|}{ Recurrence-Free Survival } \\
\hline & $\mathrm{HR}(95 \% \mathrm{Cl})$ & $P$ Value & HR $(95 \% \mathrm{Cl})$ & $P$ Value \\
\hline Anesthesia type (INHA/TIVA) & $1.303(1.065,1.595)$ & 0.010 & $1.265(1.040,1.539)$ & 0.019 \\
\hline Child-Pugh & $1.897(1.491,2.414)$ & 0.000 & $1.653(1.297,2.105)$ & 0.000 \\
\hline AFP (ug/L) & $1.099(1.010,1.194)$ & 0.027 & $1.071(0.989,1.160)$ & 0.093 \\
\hline Tumor Diameter (cm) & $1.606(1.183,2.181)$ & 0.002 & $1.492(1.123,1.983)$ & 0.006 \\
\hline PVIT & $1.160(0.989,1.360)$ & 0.068 & - & - \\
\hline
\end{tabular}


Table 3 Adverse outcomes

$\begin{array}{ll}\text { TIVA } & \text { INHA } \\ N(\%) & N(\%)\end{array}$

Dichotomous Outcomes

$\begin{array}{llll}\text { 30-day Mortality } & 4(2.1) & 11(4.7) & 0.106 \\ \text { MACE } & 4(2.1) & 11(4.7) & 0.106 \\ \text { MOD } & 6(3.3) & 9(3.9) & 0.797 \\ \text { Blood Transfusion } & 80(43.5) & 78(33.5) & 0.189 \\ & \text { Median (IQR) } & \text { Median (IQR) }\end{array}$

Continuous Outcomes

Blood Loss

$400(245,800)$

$400(300,800)$

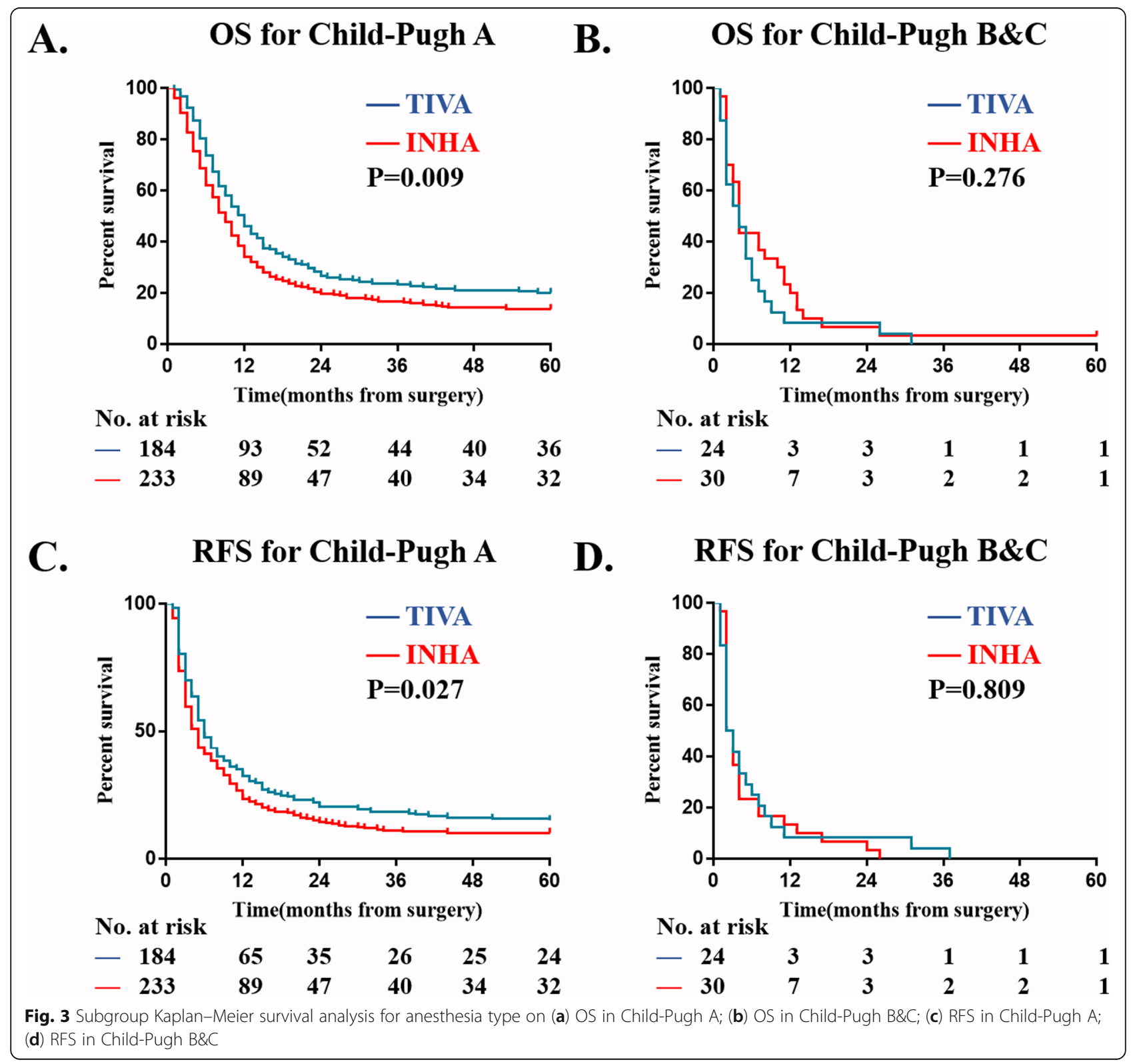




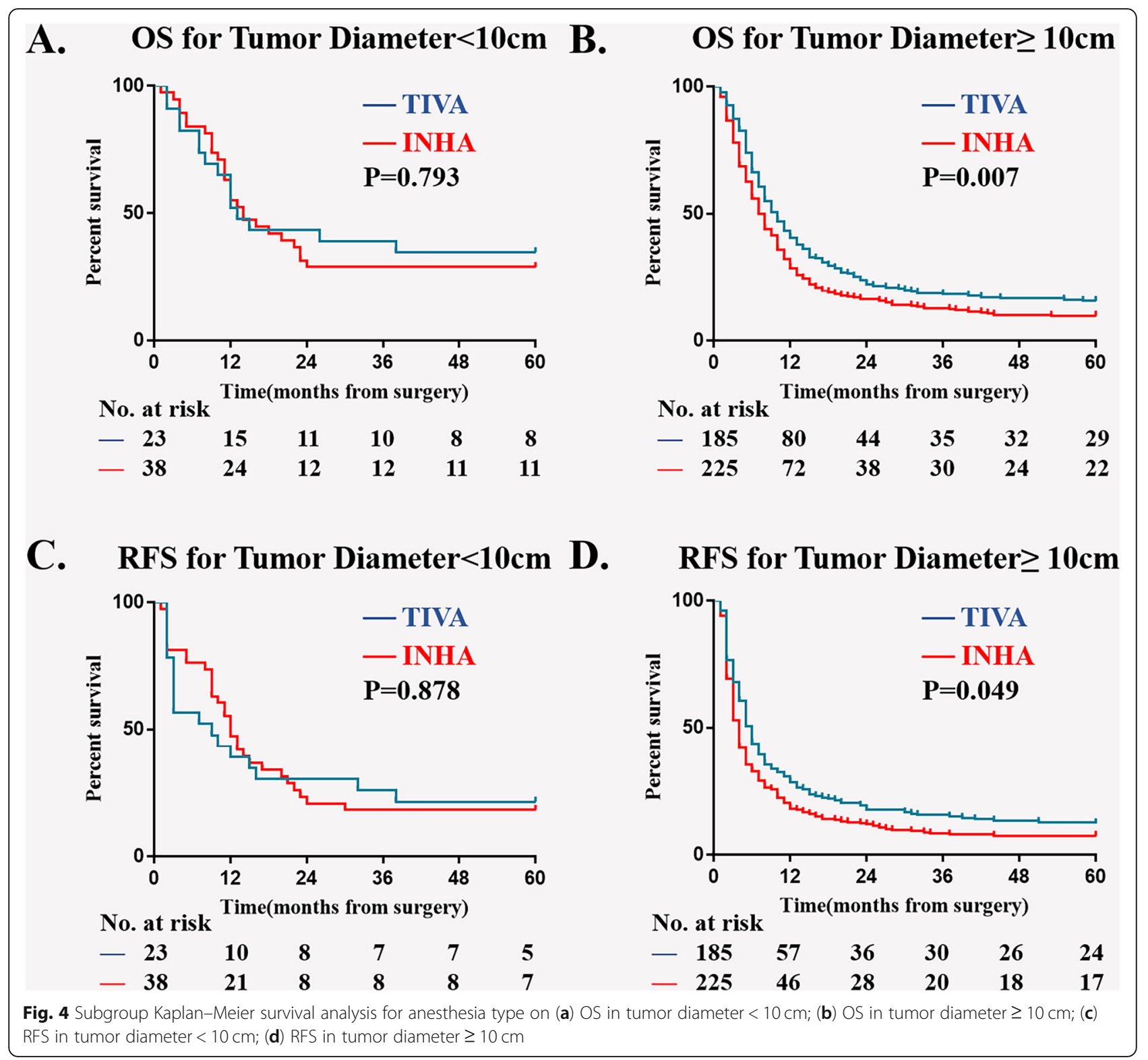

propofol, but not sevoflurane, prevents mitochondrial dysfunction and oxidative stress by limiting hypoxiainducible factor 1 alpha (HIF-1 $\alpha$ ) activation in hepatic ischemia/reperfusion injury. According to their description, this change could be beneficial for liver function, as HIF- $1 \alpha$ governs the transcription of genes controlling proliferation and metastasis of tumor cells $[17,18]$. Additionally, previous researches already demonstrate that isoflurane administration could result in an up-regulation of HIF-1 $\alpha$ in tumor [19]. However, there have no solid evidence to prove those theory in human body, while the molecular mechanisms of the different outcomes of the two anesthetic methods remains to be determined yet.

HCC with PVTT

HCC ranges as the fifth most common malignancy tumor [20]. Indeed, even worse prognosis is reported in HCC patients with PVTT, with a reported rate of $20 \%$ and a reduced median survival time (MST) of around 2-4 months compared to HCC patients without PVTT [21-24]. According to the Asia-Pacific guideline, surgery is recommended as one of the beneficial multidisciplinary treatments for PVTT. 


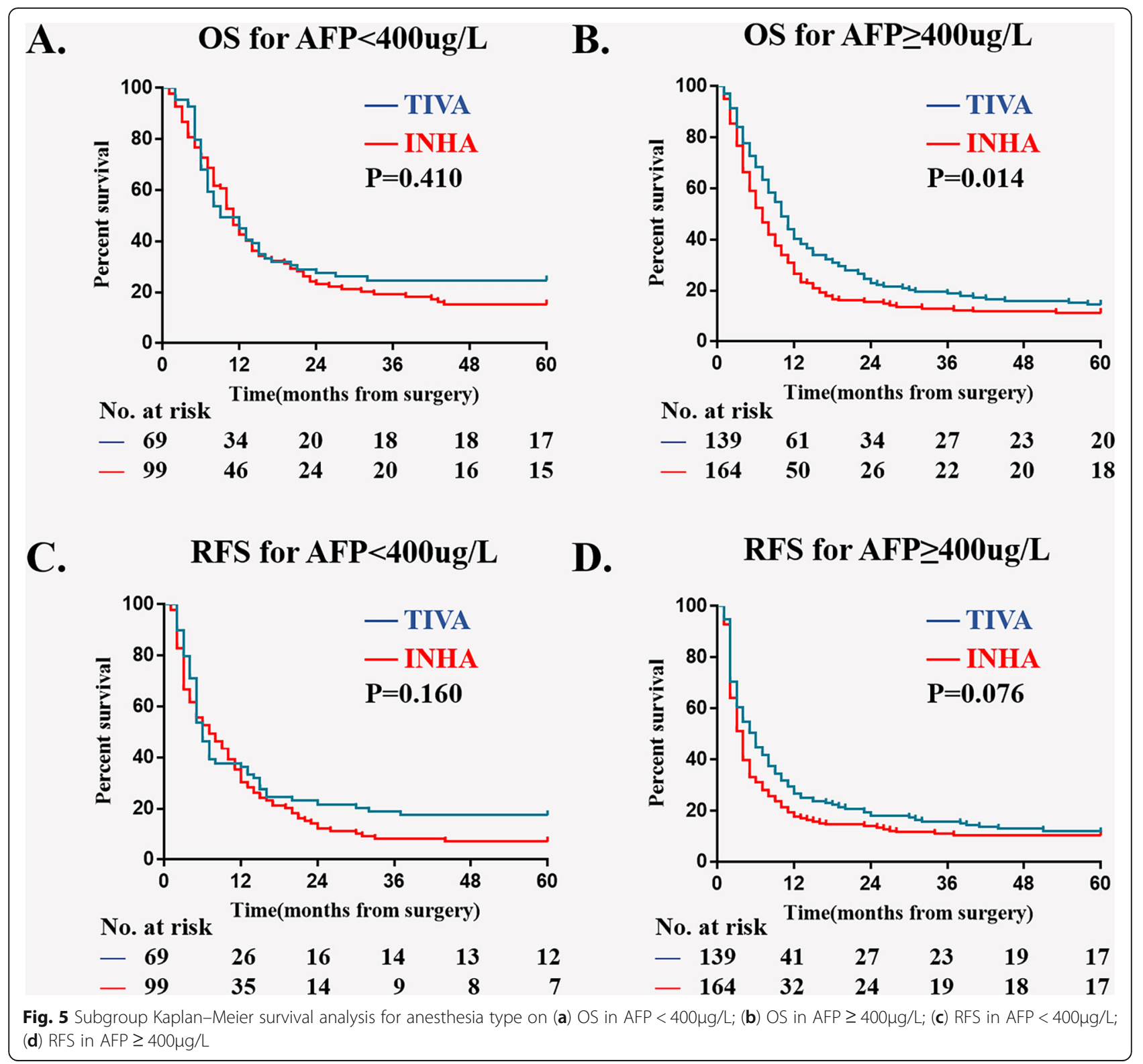

Moreover, aggressive surgical resection is associated with a longer survival outcome, and even provide chances for complete cure with type I and II PVTT $[10,25]$. Meanwhile, recent studies reported that under advanced perioperative management and skilled surgical operation, the in-hospital mortality of HCC patients with PVTT arrives an acceptable rate ranging from 3.7 to $10 \%[26,27]$. However, the knowledge about risk factors for postoperative mortality, cancer recurrence and other side events of HCC patients with PVTT still remains insufficient. Our result provides with extra evidence that anesthesia type might be a risk factor for surgical outcomes of HCC patients with PVTT.

\section{Limitations}

Several methodological discrepancies and limitations of this study should be discussed. First, there might be inclusion bias exist in our cohort, as more than a thousand patients were excluded with only 471 enrolled. Meanwhile, the majority of included patients are male, with ASA score of II, Child-Pugh score of A, and tumor size over $10 \mathrm{~cm}$. Second, certain clinical data of treatment are not collected, including perioperative chemoradiotherapy, detailed surgical techniques, and usage of opioids during surgery. Opioids have been reported to have an effect on tumor cell proliferation and angiogenesis, as well as on tumor recurrence and metastasis. However, it's hard to 


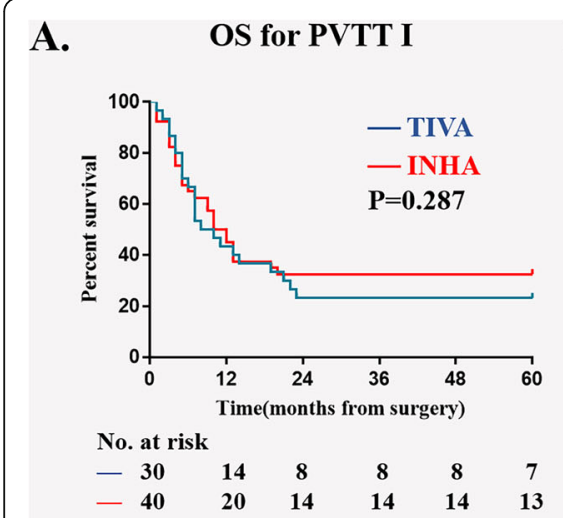

B.

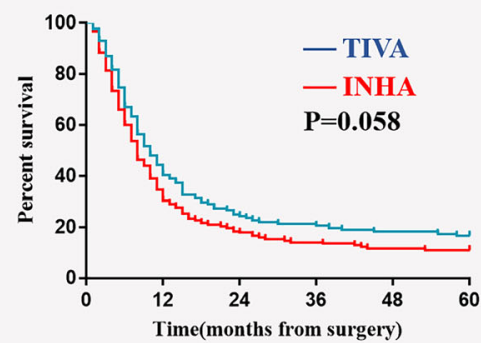

No. at risk

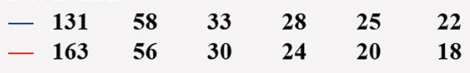

D.

OS for PVTT I

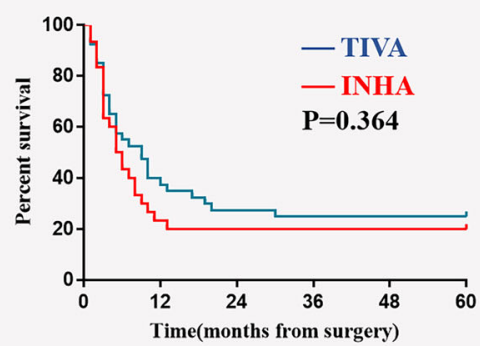

No. at risk

$\begin{array}{rrrrrr}-30 & 8 & 7 & 7 & 7 & 6\end{array}$
E.

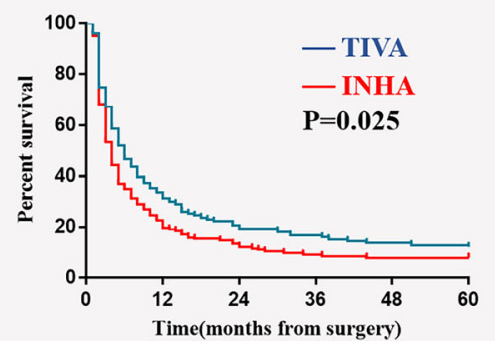

No. at risk

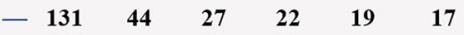

C.

OS for PVTT III

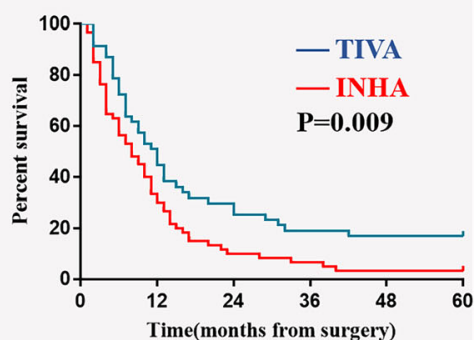

No. at risk

$\begin{array}{rrrrrr}-47 & 24 & 14 & 10 & 9 & 8 \\ -60 & 20 & 7 & 6 & 3 & 2\end{array}$

F.

OS for PVTT III

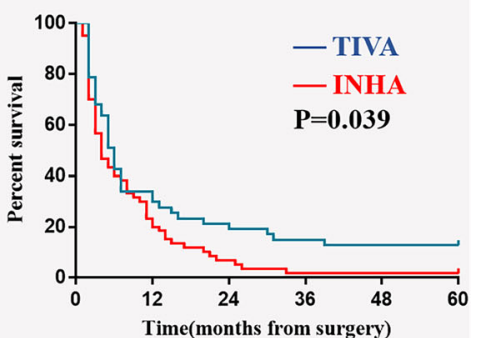

No. at risk

$\begin{array}{rrrrrr}-47 & 16 & 10 & 8 & 8 & 6\end{array}$

$\begin{array}{rrrrrr}60 & 14 & 5 & 2 & 2 & 1\end{array}$

Fig. 6 Subgroup Kaplan-Meier survival analysis for anesthesia type on (a) OS in PVTT typel; (b) OS in PVTT typell; (c) OS in PVTT typelll; (d) RFS in PVIT typel; (e) RFS in PVTT typell; (f) RFS in PVTT typelll

accurately record and compare total amounts of opioid used in both groups during surgery, as they were administered both continuously or intermittently. In this study all patients accepted at least one of remifentanil or sufentanil treatment in standard dose. Besides, since this is a retrospective analysis based clinical records and follow up data, the reason for the choice of anesthesia type at that time point, as well as potential factors affecting this choice, are not recorded or balanced. Thus, prospective researches with rigorous study design and large sample size on this field are in urgent need.

In conclusion, this retrospective analysis of longterm outcomes identifies that INHA is associated with worse survival rate compare with TIVA, and the choice of anesthesia type might be an independent risk factor for survival of HCC patients with PVTT. For some sub-variable groups (including PVTT type I, Child-puge $\mathrm{B} \& \mathrm{C}$, tumor diameter $<10 \mathrm{~cm}, \mathrm{AFP}<$ $400 \mu \mathrm{g} / \mathrm{L}$ ) there was no difference in outcomes between TIVA and INHA. Future prospective researches are urgent to verify this difference and figure out underlying causes of it.

\section{Supplementary information}

Supplementary information accompanies this paper at https://doi.org/10. 1186/s12871-020-01111-w.

Additional file 1: Table S1. Cox Proportional Hazard Regression Analyses: Univariate Model for OS and RFS.

Additional file 2.

\section{Abbreviations}

HCC: Hepatocellular carcinoma; PVIT: Portal vein tumor thrombus: INHA: Volatile inhalational anesthesia; TIVA: Total IV anesthesia; OS: Overall survival; MST: Median survival time; HIFs: Hypoxia-inducible factors; IGF: Insulin-like growth factor; MACE: Major adverse cardiac events; MOD: Multiple organ dysfunction; ALT: Alanine aminotransferase; AST: Aspartate aminotransferase; NLR: Neutrophil-lymphocyte ratio; ASA: American Society of anesthesiologists' Assessment; AFP: Alphafetoprotein

Acknowledgements

The authors thank Han Wang, M.D. for his contributions to data analysis. 


\section{Authors' contributions}

XYM helped conduct the study, analyze the data, and write the manuscript: XPZ helped conduct the study, analyze the data, ZS and HQW helped revise the manuscript; WFY helped design the study and revise the manuscript. The author(s) read and approved the final manuscript.

\section{Funding}

This study was supported by grants from the National Natural Science Foundation of China (No. 81571048); Pudong New Area Health and Family Planning Commission Joint Research Project (PW2015D-3).

\section{Availability of data and materials}

The datasets used and/or analyzed during the current study are available from the corresponding author (WF Yu; ywf808@sohu.com) on reasonable request.

\section{Ethics approval and consent to participate}

This trial had approval from the ethics committee of Eastern Hepatobiliary Surgical Hospital (EHBHHKY2012-02-028). All subjects participating in the current trial provided signed informed consent.

\section{Consent for publication}

Not applicable.

\section{Competing interests}

The authors declare that they have no competing interests.

\section{Author details}

'Department of Anesthesiology, Eastern Hepatobiliary Surgery Hospital, the Second Military Medical University, 225 Changhai Road, Shanghai, China. ${ }^{2}$ Department of Anesthesiology, Ren Ji Hospital, School of Medicine, Shanghai Jiao Tong University, 160 Pudian Road, Shanghai, China. ${ }^{3}$ Department of Hepatic Surgery, Eastern Hepatobiliary Surgery Hospital, the Second Military Medical University, 225 Changhai Road, Shanghai, China.

\section{Received: 22 September 2019 Accepted: 28 July 2020}

\section{Published online: 14 September 2020}

\section{References}

1. Wigmore TJ, Mohammed K, Jhanji S. Long-term survival for patients undergoing volatile versus IV anesthesia for cancer surgery: a retrospective analysis. Anesthesiology. 2015;60(6):240.

2. Yap A, Lopez-Olivo MA, Dubowitz J, Hiller J, Riedel B. Anesthetic technique and cancer outcomes: a meta-analysis of total intravenous versus volatile anesthesia. Can J Anaesth. 2019;66(5):546-61.

3. Huang H, Benzonana LL, Zhao H, Watts HR, Perry NJ, Bevan C, Brown R, Ma D. Prostate cancer cell malignancy via modulation of HIF-1a pathway with isoflurane and propofol alone and in combination. Br J Cancer. 2014;111(7): 1338-49.

4. Luo X, Zhao H, Hennah L, Ning J, Liu J, Tu H, Ma D. Impact of isoflurane on malignant capability of ovarian cancer in vitro f. Br J Anaesth. 2015;114(5): 831-9.

5. Fujita Y, Kimura K, Hamada H, Takaori M. Comparative effects of halothane, isoflurane, and sevoflurane on the liver with hepatic artery ligation in the beagle. Anesthesiology. 1991;75(2):313-8.

6. Enlund M, Berglund A, Andreasson K, Cicek C, Enlund A, Bergkvist L. The choice of anaestheticâ "sevoflurane or propofolâ" and outcome from cancer surgery: a retrospective analysis. Ups J Med Sci. 2014;119(3):251-61.

7. Yan T, Zhang GH, Wang BN, Sun L, Zheng H. Effects of propofol/ remifentanil-based total intravenous anesthesia versus sevoflurane-based inhalational anesthesia on the release of VEGF-C and TGF- $\beta$ and prognosis after breast cancer surgery: a prospective, randomized and controlled study. BMC Anesthesiol. 2018;18(1):131.

8. He FY, Feng WZ, Zhong J, Xu W, Shao HY, Zhang YR. Effects of propofol and dexmedetomidine anesthesia on Th1/Th2 of rat spinal cord injury. Eur Rev Med Pharmacol Sci. 2017;21(6):1355-61.

9. Kushida A, Inada T, Shingu K. Enhancement of antitumor immunity after propofol treatment in mice. Immunopharmacol Immunotoxicol. 2007;29(34):477-86.
10. Shi J, Lai ECH, Li N, Guo WX, Xue J, Wan YL, Wu MC, Cheng SQ. Surgical treatment of hepatocellular carcinoma with portal vein tumor thrombus. Ann Surg Oncol. 2010;17(8):2073-80.

11. Liu J, Dong W, Wang T, Liu L, Zhan L, Shi Y, Han J. Effects of etomidate and propofol on immune function in patients with lung adenocarcinoma. Am J Transl Res. 2016;8(12):5748.

12. Yuki K, Astrof NS, Bracken C, Soriano SG, Shimaoka M. Sevoflurane binds and allosterically blocks integrin lymphocyte function-associated antigen-1. Anesthesiology. 2010;113(113):600-9.

13. Melamed R, Bar-Yosef S, Shakhar G, Shakhar K, Ben-Eliyahu S. Suppression of natural killer cell activity and promotion of tumor metastasis by ketamine, thiopental, and halothane, but not by propofol: mediating mechanisms and prophylactic measures. Anesth Analg. 2003;97(5):1331-9.

14. Buckley A, Quaid MS, Johnson P, Buggy D. Serum from women undergoing breast cancer surgery, randomized to propofol-paravertebral anaesthetic technique, maintain natural killer cell anti-tumour activity compared with sevoflurane-opioid technique. Eur J Anaesthesiol. 2014;31:2.

15. Buckley A, Mcquaid S, Johnson P, Buggy DJ, Hemmings HC. Effect of anaesthetic technique on the natural killer cell anti-tumour activity of serum from women undergoing breast cancer surgery: a pilot study. Br J Anaesth. 2014;113(suppl 1):i56-62.

16. Bellanti F, Mirabella L, Mitarotonda D, Blonda M, Tamborra R, Cinnella G, Fersini A, Ambrosi A, Dambrosio M, Vendemiale G. Propofol but not sevoflurane prevents mitochondrial dysfunction and oxidative stress by limiting HIF-11 \pm activation in hepatic ischemia/reperfusion injury. Free Radic Biol Med. 2016;96:323-33.

17. Semenza GL. Hypoxia-inducible factors: mediators of cancer progression and targets for cancer therapy. Trends Pharmacol Sci. 2012;33(4):207-14.

18. Semenza GL. HIF-1 mediates metabolic responses to intratumoral hypoxia and oncogenic mutations. J Clin Investig. 2013;123(9):3664-71.

19. Li QF, Wang XR, Yang YW, Su DS. Up-regulation of hypoxia inducible factor 1alpha by isoflurane in Hep3B cells. Anesthesiology. 2006;105(6):1211-9.

20. Bruix J, Reig M, Sherman M. Evidence-based diagnosis, staging, and treatment of patients with hepatocellular carcinoma. Gastroenterology. 2016;150(4):835.

21. Bruix J, Sherman M. Management of hepatocellular carcinoma: an update. American association for the study of liver diseases (AASLD) practice guideline. Hepatology. 2011;53:1020-35.

22. Schöniger-Hekele M, Müller C, Kutilek M, Oesterreicher C, Ferenci P, Gangl A. Hepatocellular carcinoma in Central Europe: prognostic features and survival. Gut. 2001;48(1):103-9.

23. Balogh J, Victor D, Asham EH, Burroughs SG, Boktour M, Saharia A, Xian L, Ghobrial RM, Monsour HP. Hepatocellular carcinoma: a review. J Hepatocell Carcinoma. 2016:3:41-53.

24. Yeung YP, Lo CM, Liu CL, Wong BC, Fan ST, Wong J. Natural history of untreated nonsurgical hepatocellular carcinoma. Am J Gastroenterol. 2005; 100(9):1995-2004.

25. Kokudo T, Hasegawa K, Matsuyama Y, Takayama T, Izumi N, Kadoya M, Kudo M, Ku Y, Sakamoto M, Nakashima O. Survival benefit of liver resection for hepatocellular carcinoma associated with portal vein invasion. J Hepatol. 2016;65(5):938-43.

26. Chen JS, Wang Q, Chen XL, Huang XH, Liang LJ, Lei J, Huang JQ, Li DM, Cheng ZX. Clinicopathologic characteristics and surgical outcomes of hepatocellular carcinoma with portal vein tumor thrombosis. J Surg Res. 2012;175(2):243-50.

27. Chok KS, Cheung TT, Chan SC, Poon RT, Fan ST, Lo CM. Surgical outcomes in hepatocellular carcinoma patients with portal vein tumor thrombosis. World J Surg. 2014;38(2):490-6.

\section{Publisher's Note}

Springer Nature remains neutral with regard to jurisdictional claims in published maps and institutional affiliations. 